\title{
Visual Similarity is Greater for Line Drawings of Nonliving Than Living Things: The Importance of Musical Instruments and Body Parts
}

\author{
Keith R. Laws, ${ }^{*}$ Tim M. Gale, $\dagger+$ Ray Frank, $\dagger$ and Neil Davey $\dagger$ \\ *Department of Psychology, London Guildhall University, United Kingdom; †Departments \\ of Computer Science and Psychology, University of Hertfordshire, United Kingdom; and \\ $¥$ Queen Elizabeth Hospital, Welwyn Garden City, United Kingdom
}

\begin{abstract}
It has been argued that greater intra-category structural similarity for living things, and the subsequent "visual crowding," makes them more difficult to recognize and name for neurologically damaged individuals and normal subjects (Humphreys et al., 1988). Nevertheless, the precise meaning and quantification of structural similarity remains unclear, as does the rationale for why it necessarily should be greater for living things. We derived a new measure of visual overlap from the Snodgrass and Vanderwart corpus of line drawings: the degree of pixel overlap within subcategories (Euclidean distance: ED). Contrary to existing notions of visual crowding and extant measures of contour overlap, within-category ED indicated less within-category visual overlap for living things. Furthermore, musical instruments clustered with living things (having low overlap), while body parts clustered with nonliving things (having high overlap). These counter-intuitive findings accord with patient data and thus, provide evidence for the psychological reality and utility of ED. (c) 2002 Elsevier Science (USA)
\end{abstract}

\section{INTRODUCTION}

It has been proposed that living things (e.g. animals, fruits) have greater intracategory structural similarity or visual overlap than nonliving things (e.g., tools, vehicles); and that this visual crowding within-category makes them more difficult to name and discriminate for neurologically damaged individuals, normal subjects, and even monkeys (Humphreys, Riddoch, \& Quinlan, 1988; Gaffan \& Heywood, 1993).

In an attempt to quantify an aspect of this, Humphreys et al. (1988) developed a measure of contour overlap (CO) for subcategories (e.g., animals, clothing) of items from the Snodgrass and Vanderwart (1980) corpus of line drawings. CO was derived by overlaying a grid on each item with every other item (from the same subcategory) and then calculating the average overlap as a function of the amount of contour in each picture - overlap appears to have been matched at a gross visible level. They found that contour overlap (and hence structural similarity) was greater for living than nonliving things; and argued that this "structural similarity" may underlie categoryspecific problems with living things.

Although Humphreys and Forde (in press) refer to $\mathrm{CO}$ as an " approximate measure of the similarities of the perceptual structure of objects within their categories," it is not obvious how or why gross contour overlap alone more accurately represents structural similarity than total overlap, i.e., including internal details as well as contour. We therefore developed a measure of the total visual overlap (at pixel level) for each item with every other item within its subcategory (from the Snodgrass and Vanderwart corpus). This resulting measure of Euclidean Distance (ED) was examined to determine whether living things have greater visual overlap than nonliving things when total overlap is considered (rather than just contour overlap). It was hypothesized that ED would provide a precise measure of visual overlap between any two items: a measure of visual prototypicality, where items with lower mean EDs were more typical members of their category; and the standard deviation for an item would provide information about its relationship with other items in the comparison group. 

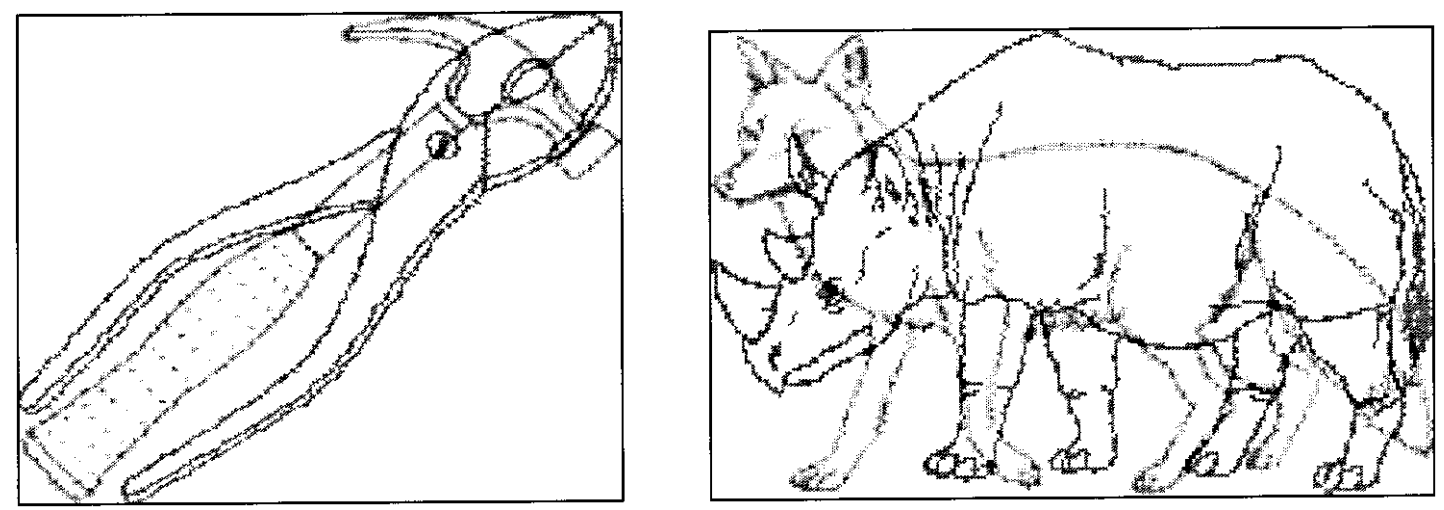

FIG. 1. Examples of overlapping figures in a nonliving (tools) and living (animals) subcategory.

\section{METHOD}

The stimuli were digitized versions of 254 images from the Snodgrass and Vanderwart (1980) line drawing corpus, scanned at a resolution of 72 dpi from the source pictures. The images were standardized for size such that the maximal dimension of each image fitted exactly within a 256 by 256 pixel grid; they were not standardized for orientation since most studies use the images in their standard presentation. The images were stored in binary bitmap format such that pixels could be either white (0) or black (1).

\section{CALCULATING EUCLIDEAN DISTANCE (ED)}

The Euclidean Distance between any two pictures was calculated by comparing the value of each pixel and then subtracting the value in the first picture from that of the second. The difference value was then squared and summed for all pixels in the array. The ED between the two pictures is the square root of the sum of squared differences (see Fig. 1). Within-category mean and standard deviation ED values were calculated for items in 6 living and 5 nonliving subcategories: animals, insects, birds, body-parts, fruit, vegetables, furniture, vehicles, musical instruments, tools, clothing (as defined by Snodgrass and Vanderwart).

\section{RESULTS}

ED correlated significantly with visual complexity $(r=.52)$, i.e., as within-category similarity increases, visual complexity decreases. CO did not correlate with ED $(r=.043)$ or with visual complexity $(r=.003)$.

Although ED did not significantly differ across living and nonliving things $(t=$ $-1.6, \mathrm{df}=130, p=.11$ : mean $=12.01[S D=1.43]$ vs $11.85[S D=1.71])$, a second analysis after removing body parts and musical instruments (which are known to be unusual categories within the living and nonliving domains) revealed significantly greater ED for living than nonliving things $(t=3.9$ [df $=118] p<.000$ : $12.3[S D=1.17]$ vs $11.41[S D=1.39])$. Moreover, these subcategories were unusual on the dimension of ED (see Fig. 2), i.e., musical instruments clustering with living things (having low overlap) and body-parts with nonliving things (having low overlap). By contrast, these two subcategories were both low in contour overlap. 

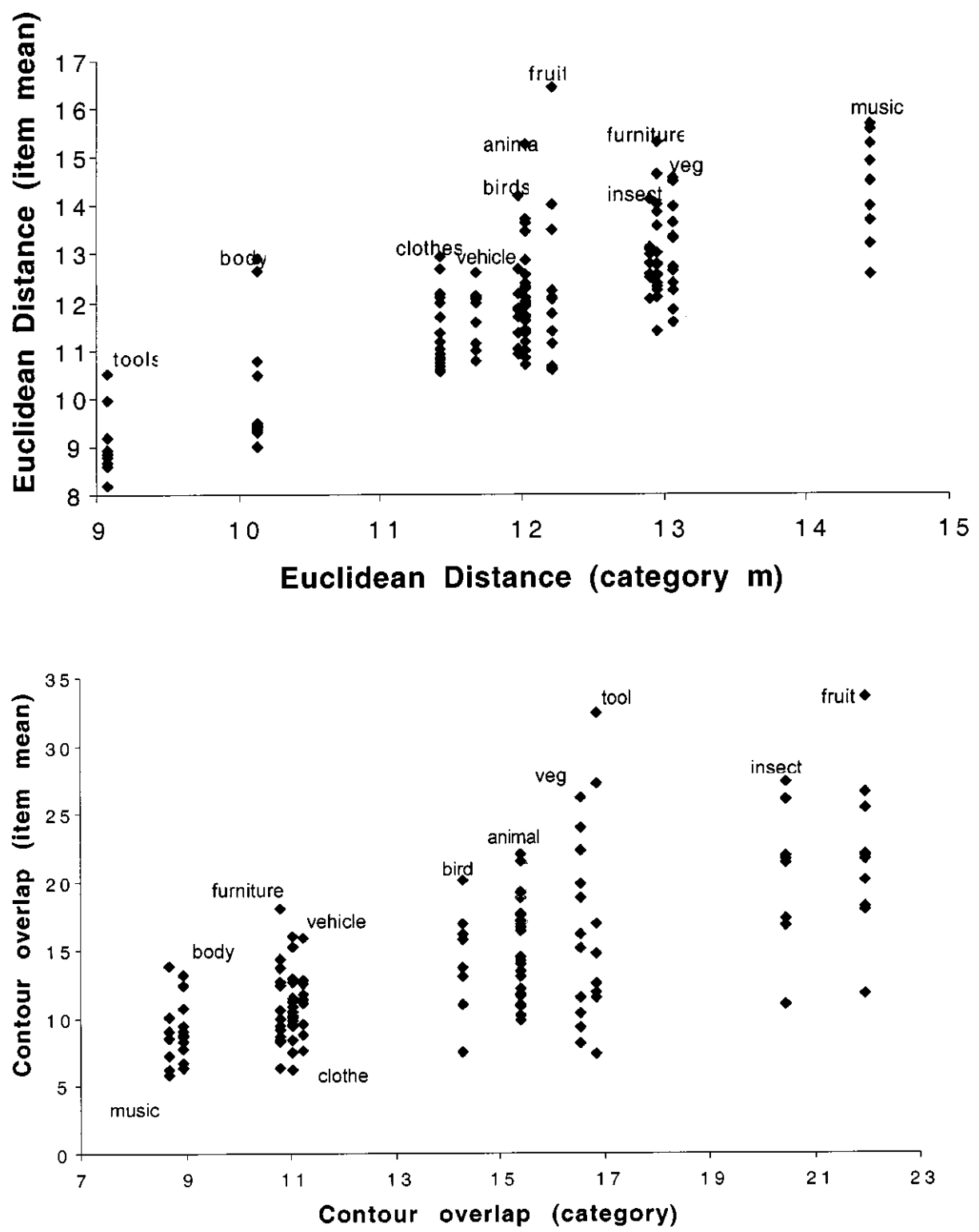

FIG. 2. Mean values for subcategories plotted against item values for Euclidean Distance and Contour Overlap.

\section{DISCUSSION}

A novel measure (ED) reflecting visual characteristics of the Snodgrass and Vanderwart corpus of line drawings was devised. Contrary to existing accounts of visual similarity/crowding and the measure of contour overlap (Humphreys et al., 1988; Gaffan \& Heywood, 1993), ED shows that nonliving things have greater withincategory visual similarity than living things. Indeed, ED was unrelated to contour overlap. The mean within-category ED for each subcategory clearly differentiated those items associated with nonliving thing disorders (having lower ED) from those associated with living thing disorders (having higher ED) ${ }^{1}$; this included musical instruments and body parts being located among living and nonliving things, respectively. Hence, these line drawings may be categorically separated at a low level of visual analysis that corresponds with the data from category-specific patients.

The pattern of results reported here are dependent upon the specific properties of

${ }^{1}$ One exception was 'furniture' which clustered among the living categories. 
the Snodgrass and Vanderwart corpus, and we would not want to suggest that these data reflect actual properties of the referent objects. Nonetheless, most investigations of category-specific deficits have utilized this corpus and so, the results have implications for the factors that purportedly contribute toward emergent category effects. For example, they might be viewed as consistent with recent findings that normal subjects have better naming of living than nonliving things from this corpus (Laws \& Neve, 1999; Laws, 1999; Laws, 2000).

One intriguing result from the analysis of ED, was that body parts and musical instruments were visually discriminable from all other subcategories (see Fig. 2); with body parts (and tools) having the greatest visual overlap and musical instruments the least. Moreover, body parts were closer in values to nonliving things, while musical instruments were closer in values to living things. This finding accords with the counter-intuitive patterns in patient data, both for living and nonliving thing cases, i.e., that musical instruments tend to be impaired along with living things and body parts with nonliving things; this finding adds weight to the psychological reality and potential utility of the measures developed here. In contrast, CO was lowest for musical instruments (8.69) and body parts (8.95). Within the model suggested by Humphreys and colleagues, living thing deficits reflect their having greater structural similarity and while this might account for why body parts are typically intact in patients with living thing disorders, it cannot incorporate the fact that musical instruments are often impaired since $\mathrm{CO}$ predicts they should remain unimpaired.

\section{ACKNOWLEDGMENTS}

The ED ratings are available from the authors on request. We thank Professor Glyn Humphreys for generously providing us with the "contour overlap" rating.

\section{REFERENCES}

Gaffan, D., \& Heywood, C. A. (1993). A spurious category-specific visual agnosia for living things in normal human and nonhuman primates. Journal of Cognitive Neuroscience, 5, 118-128.

Humphreys, G. W., Riddoch, M. J., \& Quinlan, P. T. (1988). Cascade processes in picture identification. Cognitive Neuropsychology, 5, 67-103.

Humphreys, G. W., \& Forde, E. M. E. (in press). Hierarchies, similarity and interactivity in object recognition: On the multiplicity of 'category-specific' deficits in neuropsychological populations. Behavioral and Brain Sciences.

Laws, K. R. (2000). Category-specific naming errors in normal subjects: the influence of evolution and experience. Brain and Language, 75, 123-133.

Laws, K. R. (1999). Gender affects latencies for naming living and nonliving things. Cortex, 35, 729733.

Laws, K. R., \& Neve, C. (1999). A 'normal' category-specific advantage for naming living things. Neuropsychologia, 37, 1263-1269.

Snodgrass, J. G., \& Vanderwart, M. (1980). A standardized set of 260 pictures: norms for name agreement, image agreement, familiarity and visual complexity. Journal of Experimental Psychology: Human Learning and Memory, 6, 174-215.

This is doi:10.1006/brcg.2001.1391. 